\title{
EFFECTS OF TRAFFIC CONTROL DEVICES AND ROAD SCENES ON A DRVIER'S JUDGMENT OF CURVE SHARPNESS
}

\author{
Kenta Suzuki, Kenetsu Uchida and Toru Hagiwara \\ Transportation and Traffic Engineering Laboratory \\ Hokkaido University, Sapporo, JAPAN \\ E-mail: k-suzuki@eng.hokudai.ac.jp, uchida@eng.hokudai.ac.jp, hagiwara@eng.hokudai.ac.jp
}

\author{
Takahiro Ohmi, Roberto A. Tokunaga and Motoki Asano \\ Traffic Engineering Laboratory \\ Civil Engineering Research Institute \\ Hokkaido Development Bureau \\ Sapporo, JAPAN \\ E-mail: t.oumi@ceri.go.jp, roberto@ceri.go.jp, 81105@ceri.go.jp
}

\begin{abstract}
Summary: The effects of traffic control devices and road scene on a driver's judgment of curve sharpness were investigated by field experiments on a 12-km section of rural highway running through a hilly area in Hokkaido. The configurations of traffic control devices at 36 curves were obtained from the road maintenance database. The favorability of the road scene was determined subjectively. Each of the 6 subjects drove a vehicle installed with instruments. The subject estimated the sharpness of the target curve before that curve and assessed the accuracy of that judgment subjectively after the target curve. Cluster analysis detected groupings of the 36 curves in terms of driver assessment of sharpness. The traffic control devices were verified as important cues in driver assessment of curve sharpness. In addition, the road scene had a positive effect in daytime on judgment of the target curve. However, there were some dangerous curves where the subjects underestimated the sharpness before entering the curve. Traffic control devices to provide accurate information on curve depth should be developed.
\end{abstract}

\section{INTRODUCTION}

Hagiwara, T. et al. investigated the subjective assessment of the directional detection performance of the target curve ${ }^{1,2)}$. The results indicated that the driver obtains directional information from traffic control devices at the end of tangent of the target curve and from the road scene ahead. After detecting the curve, the driver must adjust the driving speed in order to run through the target curve safety. At that time, the driver continues estimating the target curve sharpness. Usually, the curve sharpness is assessed by road alignment elements (radius, curve length, curve direction, grade, et al.). However, the driver does not know these data while driving, and can only estimate the sharpness subjectively. It is important to use cues on the road ahead to accurately estimate the curve sharpness. Cues mean traffic control devices, road scene, experience in a route and so on. Thus, we conducted a new experiment to address the following questions; how do the cues of the road scene ahead affect the estimation of the curve sharpness, what kinds of curves lead to driver misjudgment and what kinds of cues are required to minimize misjudgment? 


\section{METHODS}

\section{Experimental Site and Subjects}

The experiment was conducted on a hilly 12-km section of a two-lane rural highway in Hokkaido, Japan. The 36 curves were selected from among curves on a road section that was driven from start point to turnaround point (uphill section) and back (downhill section). The average radius was $94 \mathrm{~m}$ and $69 \mathrm{~m}$ for the uphill and the downhill section, respectively. The average gradient was $4.1 \%$ and $-4.6 \%$ for the uphill and the downhill section, respectively. Each subject drove twice in each direction on the test section: once in daytime and once at night. Six male subjects (average: 22.6 years) participated. The subjects had an average of 2.7 years of driving experience and drove an average of 15,200 km per year. Each subject had normal visual acuity, and none the subjects were familiar with the test section.

\section{Traffic Control Devices, Visual Cues and Measuring Devices}

The drivers judge sharpness of the target curve from traffic control devices and the road scene ahead. Chevron sign, guardrail, special pavement marking to warn of sharp curve ahead, sign to show the curve radius and lighting midway through the target curve were installed as cues to show the curve sharpness. A driver also uses the road scene ahead to judge sharpness of the target curve. In this study, the performance of each curve in indicating the curve sharpness was determined subjectively by several experimenters. Performance was classified according to three levels: good, medium and poor. Good means that the driver easily can judge the sharpness of the next curve due to direct view of the entire target curve ahead. Medium means that the driver can see the middle but not the end of the target curve. Poor means that the driver can see only the entrance to the target curve.

The subjects drove an experimental vehicle. A video camera installed near the rear window recorded the driving scene and the driver's voice. The driving scene included road surface conditions, traffic conditions (leading vehicle, oncoming vehicle, etc.) and a counter display. The counter display showed the pulse number generated by a transmission sensor. The pulse number represented the accumulated traveling distance from the start point as measured by transmission sensor.

\section{Experimental Design and Procedure}

One of the major dependent variables in this study was the sharpness assessment before entering the target curve, which is called the "pre-judgment". Another one was post-curve assessment of the pre- judgment, which is called the "post judgment". The pre-judgment was classified according to three sharpness levels: gentle, medium, and sharp. Post judgment was classified according to three accuracy levels: underestimation, accurate estimation and overestimation. The independent variables were the characteristics of each curve according to the configuration of traffic control devices and the favorability of road scene.

Each subject performed two runs on the test section: one in daytime and one at night. No runs were performed in rainy weather, and the road surface was always dry. At the start point, the subjects were given instructions regarding their task. The subjects were instructed to drive safely 
and to maintain a speed around $60 \mathrm{~km} / \mathrm{h}$ in the tangent section. There was never a leading vehicle. The subjects were required to judge the sharpness of each curve before and after the curve. The subjects were instructed to call their judgment of curve sharpness on the tangent section before the target curve. The experimenter gave the following explanation of the judgment criteria for curve sharpness: "'Sharp' means that you are required to slow down before entering the target curve. 'Medium' means that you are required to brake slightly before entering the target curve. 'Gentle' means that you can run through the target curve safely without slowing down." The subjects evaluated their pre-judgment as soon as possible after the target curve.

"Underestimation" means that the passed curve was sharper than predicted. The subject might be required to slow down in order to run through the target curve safety. "Accurate estimation" means that the passed curve was about as sharp as predicted. "Overestimation" means that the passed curve was gentler than predicted. After finishing the run, each subject was asked several questions concerning the judgment of the curve.

\section{RESULTS}

\section{Pre- and Post Judgment and Driving Speeds}

Six data were recorded at each curve. Integers from 1 to 3 were assigned to the three pre-judgments of sharpness, with 3 for the sharpest curve. Integers from 1 to 3 were assigned to the three subjective post judgments of sharpness, with 2.0 indicating perfect correspondence between pre- and post judgement. A post judgment larger than 2.0 indicated a sharper curve than predicted, a situation that might lead to an accident. A post judgment of less than 2.0 indicated a gentler curve than predicted. Uphill, the average pre-judgment was 2.3 in daytime and 2.3 at night. Downhill, it was 2.5 in daytime and 2.4 at night. The average post judgment was 2.1 in daytime and 2.1 at night, both for uphill. For downhill, it was 2.3 in daytime and 2.2 at night. Pre-judgment both in daytime and at night were about the same for uphill and downhill. The subjects evaluated the curves as sharper when traveling downhill than when traveling uphill. The pre-judgments for downhill exceeded those for uphill at both nighttime and daytime, and the post judgments downhill exceeded 2.0, which exceeded those for uphill. In addition, both the pre-curve speed and the curve speed in daytime were about $4 \mathrm{~km}$ less than those at night.

Three driving speeds were calculated using recorded data. One was the speed before entering the target curve ("pre-curve speed"). The pre-curve speed was measured at $10 \mathrm{~m}$ ahead of the beginning of the target curve. Another was the average speed on the target curve between the beginning and the end of the target curve ("curve speed"). The third was difference between the pre-curve speed and the curve speed ("speed difference). Table 1 shows the pre-curve speeds, the curve speeds and the speed difference for each section. In daytime, these three speeds were about the same in both sections. The pre-curve speed was about $55 \mathrm{~km} / \mathrm{h}$, the curve speed was about $49 \mathrm{~km} / \mathrm{h}$ and the speed difference was about $6 \mathrm{~km} / \mathrm{h}$. At night, for both uphill and downhill, the pre-curve speed was $52 \mathrm{~km} / \mathrm{h}$ and the curve speed was $46 \mathrm{~km} / \mathrm{h}$. However, the speed difference of downhill section at nigh was $6.3 \mathrm{~km} / \mathrm{h}$, which was the greatest speed difference of the four cases.

\section{Cluster Analysis}

Cluster analysis detected groupings of 17 curves on the uphill section and 18 curves on the downhill section. The data of pre-judgment scores and post judgment scores in daytime and at night were clustered. A dendrogram was used to assess the characteristics of grouped curves in 
cluster formation. Figure 1 shows the pre-judgment scores, the post judgment scores and the speed difference for eight groups, respectively. Also, Table 2 shows the average values of road alignment elements, the percentages of curves installed with traffic control devices, and the favorability of the road scene.

TABLE 1. Road alignment elements, driving speeds and subjective assessments for the two sections

\begin{tabular}{|c|c|c|c|c|c|c|c|c|c|c|c|c|c|c|}
\hline & & \multicolumn{3}{|c|}{ Road Alignment } & \multicolumn{6}{|c|}{ Driving speed } & \multicolumn{4}{|c|}{ Subjective assessment } \\
\hline & & \multirow[b]{2}{*}{$\begin{array}{c}\text { Radius } \\
\text { (m) }\end{array}$} & \multirow[b]{2}{*}{$\begin{array}{c}\text { Curve } \\
\text { Length } \\
\text { (m) }\end{array}$} & \multirow[b]{2}{*}{$\begin{array}{c}\text { Grade } \\
(\%)\end{array}$} & \multicolumn{3}{|c|}{ Day } & \multicolumn{3}{|c|}{ Night } & \multicolumn{2}{|c|}{ Day } & \multicolumn{2}{|c|}{ Night } \\
\hline & & & & & $\begin{array}{l}\text { Pre- } \\
\text { curve } \\
(\mathrm{km} / \mathrm{h})\end{array}$ & $\begin{array}{l}\text { Curve } \\
(\mathrm{km} / \mathrm{h})\end{array}$ & $\begin{array}{c}\text { Differ- } \\
\text { ence } \\
(\mathrm{km} / \mathrm{h})\end{array}$ & $\begin{array}{c}\text { Pre- } \\
\text { curve } \\
(\mathrm{km} / \mathrm{h})\end{array}$ & $\begin{array}{l}\text { Curve } \\
(\mathrm{km} / \mathrm{h})\end{array}$ & $\begin{array}{l}\text { Differ- } \\
\text { ence } \\
(\mathrm{km} / \mathrm{h})\end{array}$ & Pre & Post & Pre & Post \\
\hline \multirow{3}{*}{$\begin{array}{l}\text { Uphill } \\
\text { section }\end{array}$} & average & 94 & 123 & 4.1 & 55.6 & 49.8 & 5.8 & 51.3 & 46.1 & 5.2 & 2.3 & 2.1 & 2.3 & 2.1 \\
\hline & std. & 97 & 37 & 2.0 & 4.1 & 6.2 & 5.1 & 4.3 & 6.1 & 3.8 & 0.4 & 0.3 & 0.5 & 0.2 \\
\hline & $\mathrm{n}$ & 17 & 17 & 17 & 102 & 102 & 102 & 102 & 102 & 102 & 102 & 100 & 101 & 98 \\
\hline \multirow{3}{*}{$\begin{array}{l}\text { Downhill } \\
\text { section }\end{array}$} & average & 69 & 125 & -4.6 & 54.4 & 48.9 & 5.5 & 52.2 & 45.9 & 6.3 & 2.5 & 2.3 & 2.4 & 2.2 \\
\hline & std. & 32 & 49 & 1.5 & 5.4 & 5.8 & 4.8 & 4.5 & 5.2 & 3.9 & 0.4 & 0.3 & 0.4 & 0.2 \\
\hline & $\mathrm{n}$ & 19 & 19 & 19 & 114 & 114 & 114 & 114 & 114 & 114 & 114 & 114 & 114 & 113 \\
\hline
\end{tabular}

(A) Uphill
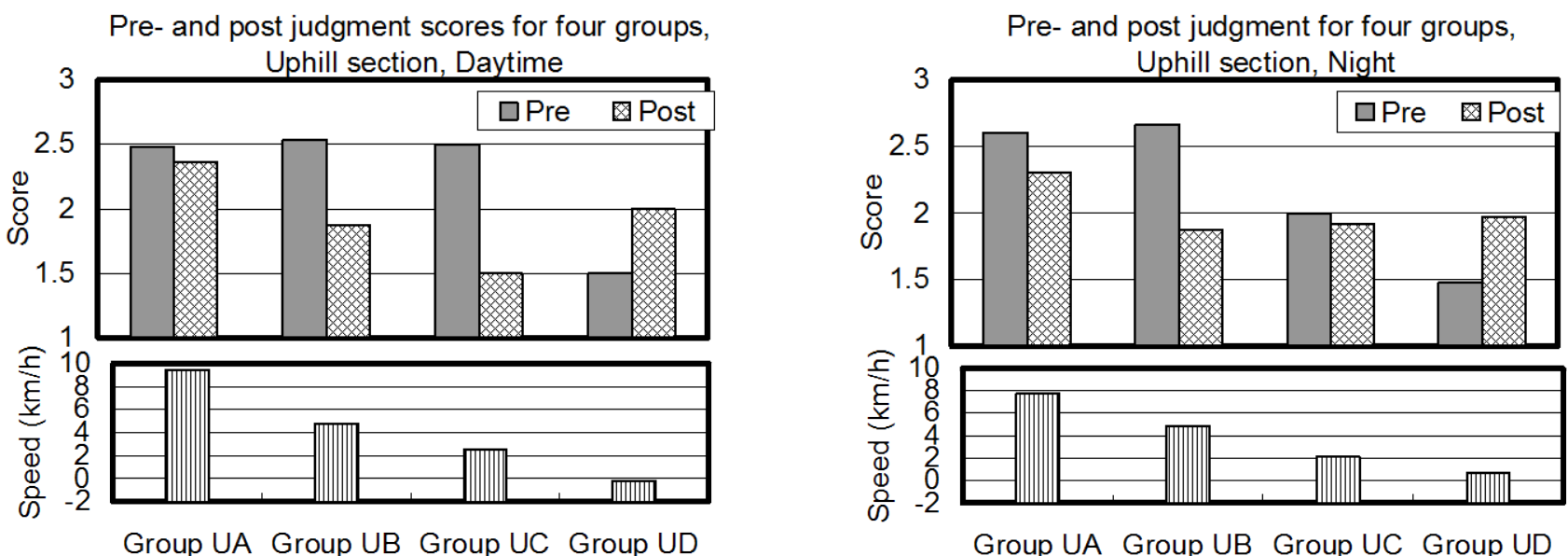

(B) Downhill

Pre- and post judgment scores for four groups,

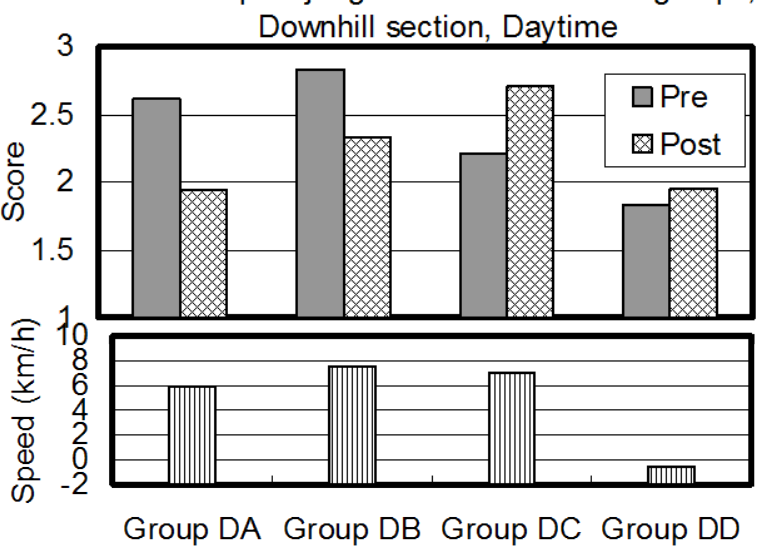

Pre- and post judgment scores for four group,

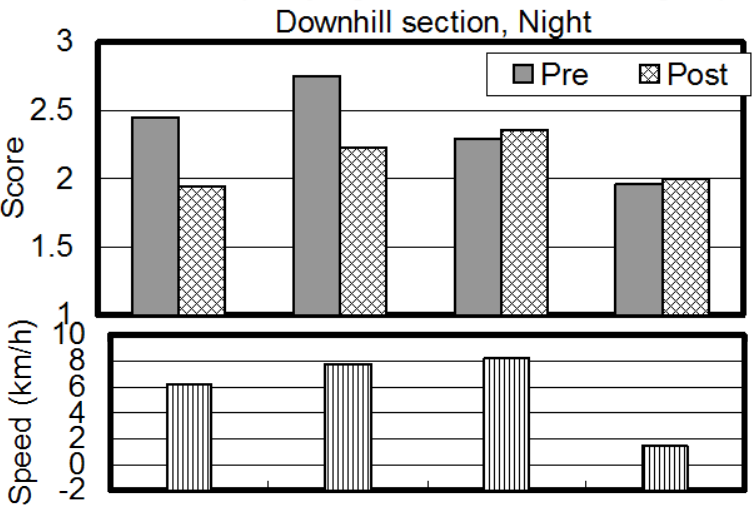

Group DA Group DB Group DC Group DD

Figure 1. Average pre- and post judgment scores and average speed differences for eight groups in daytime and at night. 
Uphill section. Group UA: Pre-judgment scores exceeded 2.5, and post judgment scores exceeded 2.0. Typical curves in Group UA had small radii, neutral road scene and less traffic control devices than Group UB had. The speed differences in daytime and at night were the largest of the eight groups. Group UB: Pre-judgment scores exceeded 2.5, but post judgment scores were close to 2.0. Curve alignment was similar to that of Group UA. Group UB had rich traffic control devices and an unfavorable road view. Group UC: Pre-judgment scores exceeded 2.0 but were less than those of Group UA and Group UB. The road alignments were more favorable than those in Group UA and Group UB. The post judgment scores were under 2.0. These curves had no chevron signs and the road view was moderately unfavorable. Group UD: Pre-judgment scores were around 2.0, and post judgment scores were only 2.0. These curves had a favorable road view, large radii, and were less well endowed with traffic control devices. The speed difference was approximately $0 \mathrm{~km} / \mathrm{h}$.

Downhill section. Group DA: Pre-judgment scores were around 2.5, and post judgment scores were close to 2.0. Curve alignments were not unfavorable compared with the other groups, and these curves were well endowed with traffic control devices. Group DB: Pre-judgment scores exceeded 2.5 and values were the largest of the eight groups. In addition, post judgment scores exceeded 2.0. Typical of the curves in Group DB were small radii and relatively great curve length. The road view was moderately unfavorable and most of these curves were well endowed with traffic control devices The speed differences at both times were about $8 \mathrm{~km} / \mathrm{h}$. Group DC: Pre-judgment scores were not so large; however, the post judgment scores were the largest of the eight groups. The road alignments were about the same as those in Group DB. Three curves had no chevron signs, and the road scene was moderately favorable. The speed difference at night was $8.2 \mathrm{~km} / \mathrm{h}$. Group DD: Pre-judgment scores were about 2.0, and post judgment scores were exactly 2.0. These curves had a favorable road view, large radii, and were less well endowed with traffic control devices. In addition, the speed difference was approximately $0 \mathrm{~km} / \mathrm{h}$.

TABLE 2. Average values of the road alignment elements and the percentages of curves installed with traffic control devices, per group, for eight groups.

\begin{tabular}{|c|c|c|c|c|c|c|c|c|c|}
\hline \multicolumn{3}{|c|}{ (A) Uphill section } & \multirow{2}{*}{$\begin{array}{l}\text { Curve } \\
\text { length } \\
(\mathrm{m}) \\
\end{array}$} & \multirow{2}{*}{$\begin{array}{c}\text { Clothoid } \\
\text { curve } \\
(\%)\end{array}$} & \multirow{2}{*}{$\begin{array}{c}\text { Chevron } \\
\text { sign } \\
(\%) \\
\end{array}$} & \multirow{2}{*}{$\begin{array}{c}\text { Warning } \\
\text { marking } \\
(\%)\end{array}$} & \multirow{2}{*}{$\begin{array}{l}\text { Road } \\
\text { lighting } \\
(\%)\end{array}$} & \multirow{2}{*}{$\begin{array}{l}\text { Sign of } \\
\text { radius } \\
(\%)\end{array}$} & \multirow{2}{*}{$\begin{array}{l}\text { Road } \\
\text { scene } \\
\text { (score) }\end{array}$} \\
\hline Group & $\begin{array}{l}\text { Number } \\
\text { of curves }\end{array}$ & $\begin{array}{c}\text { Radius } \\
(\mathrm{m})\end{array}$ & & & & & & & \\
\hline UA & 8 & 48 & 137 & 37.5 & 37.5 & 12.5 & 75 & 100 & 2 \\
\hline UB & 4 & 56 & 97 & 50 & 75 & 50 & 50 & 100 & 1 \\
\hline UC & 2 & 95 & 122 & 50 & 0 & 50 & 50 & 100 & 1.5 \\
\hline UD & 3 & 267 & 121 & 33 & 0 & 33 & 33 & 33 & 3 \\
\hline \multicolumn{3}{|c|}{ (B) Downhill section } & Curve & Clothoid & Chevron & Warning & Road & Sign of & Road \\
\hline Group & $\begin{array}{l}\text { Number } \\
\text { of curves }\end{array}$ & $\begin{array}{c}\text { Radius } \\
\text { (m) }\end{array}$ & $\begin{array}{l}\text { length } \\
\text { (m) }\end{array}$ & $\begin{array}{c}\text { curve } \\
(\%)\end{array}$ & $\begin{array}{l}\text { sign } \\
(\%)\end{array}$ & $\begin{array}{c}\text { marking } \\
(\%)\end{array}$ & $\begin{array}{c}\text { lighting } \\
(\%)\end{array}$ & $\begin{array}{c}\text { radius } \\
(\%)\end{array}$ & $\begin{array}{l}\text { scene } \\
\text { (score) }\end{array}$ \\
\hline $\mathrm{DA}$ & 3 & 73 & 87 & 33 & 100 & 100 & 67 & 67 & 2 \\
\hline DB & 8 & 47 & 132 & 37.5 & 62.5 & 37.5 & 62.5 & 87.5 & 1.4 \\
\hline DC & 4 & 64 & 153 & 25 & 25 & 50 & 100 & 100 & 1.8 \\
\hline DD & 4 & 114 & 111 & 50 & 0 & 0 & 25 & 50 & 2.5 \\
\hline
\end{tabular}




\section{DISCUSSION AND CONCLUSIONS}

This study investigated the qualitative relationships between cues and assessment of curve sharpness in daytime and at night. The subjects assessed 36 curves. The 17 uphill curves were classified into four groups using the results of the subjects' assessment, and the 19 downhill curves were classified into four groups. Analysis of the effects of cues on the pre-judgment and post judgment of curve sharpness found that Group DB, Group DC and Group UA are potentially dangerous curves. The other five groups are less dangerous, because drivers are able to make appropriate post judgments of them. In Group DC, the pre-judgment scores were not so large; however, the post judgment scores were the largest of the eight groups. The post judgment scores in daytime were particularly large. The main reason was lack of the traffic control devices accompanied by moderately unfavorable road scene and long curve length. These curves may therefore be considered potentially dangerous, and should be installed with traffic control devices that show the curve depth in daytime. Group UA was similar to Group DB. These groups had high pre-judgment scores and high post judgment scores, due to the short radius and the relatively long curve length. In these curves, the drivers noticed the sharp curve ahead, and reduced their speed. However, notification or warning to drivers was insufficient in allowing them to run through the target curve safely. The driver assessed that the passed curve was sharper than was predicted before entering the next target curve. These discussions show that traffic control devices significantly affect the pre-judgment score but these alone cannot provide complete information on the curve alignment. We should consider how to inform curve depth to the driver. In addition, it should be noted that the criteria of pre- and post judgment were not so clear and relationships between both subjective judgments and a number of accidents were not discussed. These call for further investigations of various curves and conditions.

\section{References}

1) Hagiwara, T., Suzuki, K., Tokunaga, R.A., Kagaya, S. and Onuma, H., Effects of landscape and Traffic Control Devices on a Driver's Curve Detection Performance in the Daytime and at Night, Paper No.00-1481 presented at the 79 TRB Annual Meeting, 2000.

2) Hagiwara, T., Suzuki, K., Tokunaga, R.A., Yorozu, N. and Asano, M., Field Study of a Driver's Curve Detection Performance in the Daytime and at Night, Paper No.00-3075 presented at the 80 TRB Annual Meeting, 2001. 